\title{
Pulmonary hypertension in hypoventilation syndromes
}

\author{
Robert Naeije \\ Affiliation: Dept of Pathophysiology, Erasme Academic Hospital, Free University of Brussels, Brussels, \\ Belgium.
}

Correspondence: R. Naeije, Dept of Physiology, Erasme Campus CP 604, 808 Lennik road, B-1070 Brussels, Belgium. E-mail: rnaeijedulb.ac.be

The object that presented itself to the eyes of the astonished clerk, was a boy - a wonderfully fat boy - habited as a serving lad, standing upright on the mat, with his eyes closed as if in sleep.

- Dickens [1]

In this issue of the European Respiratory Journal, HeLD et al. [2] report on 18 patients with severe pulmonary hypertension due to alveolar hypoventilation, who were markedly improved after 3 months of noninvasive bi-level positive-pressure ventilation (NIPPV). Mean pulmonary artery pressure (mPAP) and pulmonary vascular resistance (PVR) were $50 \mathrm{mmHg}$ and 6-7 Wood units, respectively, at baseline, and decreased to $30 \mathrm{mmHg}$ and 3-4 Wood units with NIPPV; this was accompanied by improved catherisation and echocardiographic indices of right ventricular (RV) function. The N-terminal pro-brain natriuretic peptide (NT-proBNP) levels also decreased, and the 6-min walk distance (6MWD) improved by $80 \mathrm{~m}$. These results are in striking contrast with the "borderline" (mPAP 20-25 mmHg) or mild (mPAP 25-30 mmHg) pulmonary hypertension generally seen, but with rare exceptions, in patients with respiratory conditions. Borderline or mild pulmonary hypertension due to respiratory conditions is of uncertain clinical relevance, exhibits slow progression controlled by supplemental oxygen and is refractory to drugs targeting the pulmonary circulation $[3,4]$.

12 of the 18 patients reported by HeLD et al. [2] fulfilled the criteria of obesity hypoventilation syndrome (OHS), defined by a combination of awake hypercapnia (arterial carbon dioxide tension $\left(\mathrm{PaCO}_{2}\right)$ $>45 \mathrm{mmHg}$ ), a body mass index $>30 \mathrm{~kg} \cdot \mathrm{m}^{-2}$ and exclusion of other causes that could account for hypoventilation, such as lung or neuromuscular disease $[5,6]$. Three of the OHS patients had concomitant chronic obstructive pulmonary disease (COPD) and five patients had COPD without obesity. However, the reported patient population may be representative of OHS as sleep disordered breathing with obstructive sleep apnoea (OSA) is identified in $90 \%$ of the patients and OSA often presents as "overlap syndrome", defined by concomitant COPD $[5,6]$.

OHS was first reported in 1955 [7] and, a year later, the term Pickwickian syndrome was coined [8], after the colourful character of the "fat boy" who consumes great quantities of food and constantly falls asleep in any situation at any time of day, described by Charles Dickens in The Pickwick Papers. But patients afflicted with OHS do not find it funny. They suffer from fatigue, exertional dyspnoea, morning headaches, daytime sleepiness and depression, impacting both the physical and mental/social aspects of quality of life. In addition, they are more exposed than obese control subjects to comorbidities, including hypertension, heart failure, pulmonary hypertension, asthma and diabetes mellitus, resulting in higher medical resource utilisation and shorter life expectancy $[5,6]$. The pathophysiological changes in OHS include an excessive

@ERSpublications

"Treat the lung not the pressure" is the strategy for patients with pulmonary hypertension and respiratory conditions http://ow.ly/qso8o

Received: Oct 232013 | Accepted after revision: Oct 242013

Conflict of interest: None declared.

Copyright @ERS 2014 
mechanical load imposed on the respiratory system by increased weight, a blunted central respiratory drive (which is normally increased in overweight control subjects) and sleep disordered breathing. There are several associated biological disturbances, including decreased leptin and insulin-like growth factor-1, both of which correlate to increased $\mathrm{PaCO}_{2}$. The incidence of OHS is increasing along with the rising incidence of extreme obesity around the world, and can, therefore, be considered a rapidly growing public health problem. Current estimates suggest that OHS might be diagnosed in $0.3-0.4 \%$ of the US population $[5,6]$.

Up to $50 \%$ of patients with OHS present with peripheral oedema, pulmonary hypertension and right heart failure, as compared with a small percentage of patients with "pure" OSA [5]. However, the severity of pulmonary hypertension in OHS is variable. For example, an average PVR of 4 Wood units and mPAP of $23 \mathrm{mmHg}$ was observed in a series of 27 patients with OHS by KessLer et al. [9], contrasting with much higher PAP and PVR in the study of HELD et al. [2]. These differences probably reflect variable referral strategies in different communities and the wide range of pulmonary vascular responses to hypercapnia and hypoxia. Whether OHS would be a more common cause of severe pulmonary hypertension than other respiratory conditions remains to be firmly established.

The reversibility of pulmonary hypertension in OHS treated with NIPPV suggests a major pathological role for hypoxic and hypercapnic vasoconstriction. In 1971, HARVEY et al. [10] proposed the following equation, elaborated on available data at that time, to predict diastolic PAP (dPAP) from wedged PAP (wPAP), arterial oxygen saturation $\left(\mathrm{SaO}_{2}\right)$ and hydrogen ion concentration $\left[\mathrm{H}^{+}\right]$in patients with respiratory conditions:

$$
\mathrm{dPAP}=-15.4+(0.63 \times \mathrm{wPAP})+\left(0.58 \times \mathrm{SaO}_{2}\right)+\left(0.56 \times\left[\mathrm{H}^{+}\right]\right)
$$

The authors reasoned that the dPAP-wPAP gradient, or diastolic pulmonary vascular pressure gradient (DPG), would be a better indicator of pulmonary vascular disease than PVR or the MPAP-wPAP gradient, or transpulmonary pressure gradient, because mPAP, not dPAP, is affected by pulmonary arterial compliance and stroke volume [10]. This has been recently revisited and confirmed [11]. HARVEY et al. [10] also thought that the effects of increased $\mathrm{PaCO}_{2}$ on the pulmonary circulation are mediated by a decreased $\mathrm{pH}$, as shown by animal experiments [12] and also more recently confirmed [13]. It is fascinating that the DPG values before and after NIPPV reported in the study by HeLD et al. [2] are predicted by Harvey's formula.

While the improvement in haemodynamics after 3 months of NIPPV was striking [2], with (quasi)normalisation of most noninvasive and invasive indices of RV function, NT-proBNP and PVR, pulmonary hypertension was not completely reversed. This may be related to incomplete correction of daytime hypercapnia, which remained, on average, at the extreme upper limit of normal or above, and one does not have information about night-time hypercapnia. The authors could have thought of using a measurement of bicarbonate, which remains persistently increased after nocturnal bouts of hypercapnia [5]. Erythrocytosis, which is another important feature of the OHS, was not mentioned but probably corrected by NIPPV. Vascular resistance is directly affected by the viscosity of the blood. In patients with chronic mountain sickness (CMS), exposed to severe hypoxaemia at high altitude related to a blunting of chemosensitivity to hypoxia, correction of PVR for the effects of increased haematocrit decreases PVR by an average of 2-3 Wood units [14]. Abnormal respiratory mechanics of obesity and associated COPD could be causes for persistently increased PVR in a proportion of the patients. Concomitant weight loss would probably have further increased the efficacy of NIPPV $[5,6]$. However, weight loss is very difficult to achieve in OHS using a medical approach. Bariatic surgery is more effective, but not always successful and carries a high risk of serious postoperative complications [4-6]. Finally, longer periods of NIPPV may be needed to completely reverse the hypoxic remodelling of the pulmonary circulation. Normalised pulmonary haemodynamics in CMS, the altitude counterpart of OHS, has been reported to require 1-2 years of sea level residency [15].

Hypercapnia is well tolerated in critically ill patients mechanically ventilated with small tidal volumes to prevent inflation-induced parenchymal lung injury [16]. However, hypercapnia in patients living with a hypoventilation syndrome is a curse. The retention of carbon dioxide causes dyspnoea-fatigue symptoms, poor sleep, morning headaches, daytime somnolence, flapping tremor and confusion, all making daily life miserable [17]. Increased carbon dioxide tension alters central nervous system function, has direct negative effects on myocardial contractility, decreases muscle strength and induces systemic vasodilation which, along with associated sympathetic activation, results in a hyperdynamic cardiovascular state [17]. Renal function adapts by an increased reabsorption of bicarbonate, but this is necessarily accompanied by retention of sodium [18] and, thus, fluid retention and eventual congestion. Associated metabolic alkalosis further depresses the central chemoreflex in a vicious circle. 
Why do patients with OHS present with a decreased exercise capacity? HeLD et al. [2] suggest that pulmonary hypertension plays a major role, as decreased PAP and improved 6MWD as a result of NIPPV were correlated. This was also observed by CASTRO-AÑón et al. [19] in a similar study in 30 patients with OHS treated with NIPPV for 6 months, but with pulmonary hypertension assessed noninvasively. However, factors other than the unloading of the RV could also positively affect the 6MWD, including improved oxygenation increasing oxygen delivery to the working muscles and improved cerebral function by correction of hypercapnic acidosis. Cardiopulmonary exercise testing was performed in nine of the patients reported by HELD et al. [2], but with unclear results as the maximum respiratory exchange ratio remained below one, excluding an early anaerobic threshold like that typically seen in heart failure or pulmonary hypertension [20, 21]. Preserved breathing reserve (that is the difference between maximum voluntary ventilation and maximum exercise ventilation) argues against a ventilatory limitation, as was also shown in CMS [22]. Further studies are needed to better understand the contribution of pulmonary hypertension to the limitation of exercise capacity in OHS.

HeLD et al. [2] are to be commended for this elegant pathophysiological study. Their results shed light on the multiple metabolic and cardiorespiratory derangements accounting for the clinical presentation of OHS. As with any good study, new questions arise that will hopefully encourage further research. The authors raise the possibility that severe pulmonary hypertension in hypoventilation syndromes may curable by simple and straightforward measures aimed at correction of respiratory disturbances.

In 1990, after several years of negative reports of the effects of vasodilators, including $\beta_{2}$-agonists, hydralazine, isosorbide, calcium antagonists and angiotensin converting enzyme inhibitors in patients with pulmonary hypertension on respiratory conditions, PEACOCK [23] called for a break and urged colleagues to "Treat the lung not the [pulmonary artery] pressure". He advised referral of these patients to specialist units for optimised treatment with judicious use of oxygen, bronchodilators, steroids, ventilatory support and dietary counselling as needed. Almost a quarter of a century later this has not changed, in spite of the advent of targeted therapies efficacious in the treatment of pulmonary arterial hypertension.

The study by HELD et al. [2] is a reminder that "treating the lung, not the pressure" is currently the recommended therapeutic strategy for patients with pulmonary hypertension and respiratory conditions.

\section{References}

Dickens C. The Posthumous Papers of the Pickwick Club. London, Chapman and Hall, 1836.

Held M, Walthelm J, Baron S, et al. Functional impact of pulmonary hypertension due to hypoventilation and changes under noninvasive ventilation. Eur Respir J 2014; 43: 156-165.

3 Hoeper MM, Barberà JA, Channick RN, et al. Diagnosis, assessment, and treatment of non-pulmonary arterial hypertension pulmonary hypertension. J Am Coll Cardiol 2009; 54: Suppl. 1, S85-S96.

Han MK, McLaughlin VV, Criner GJ, et al. Pulmonary diseases and the heart. Circulation 2007; 116: 2992-3005. Mokhlesi B, Tulaimat A. Recent advances in obesity hypoventilation syndrome. Chest 2007; 132: 1322-1336.

Piper AJ, Grunstein RR. Obesity hypoventilation syndrome: mechanisms and management. Am J Respir Crit Care Med 2011; 183: 292-298.

7 Auchinloss JH Jr, Cook E, Renzetti AD. Clinical and physiological aspects of a case of obesity, polycythemia and alveolar hypoventilation. J Clin Invest 1955; 34: 1537-1545.

8 Bickelmann AG, Burwell CS, Robin ED, et al. Extreme obesity associated with alveolar hypoventilation: a Pickwickian syndrome. Am J Med 1956; 21: 811-818.

9 Kessler R, Chaouat A, Schinkewitch P, et al. The obesity-hypoventilation syndrome revisited: a prospective study of 34 consecutive cases. Chest 2001; 120: 369-376.

10 Harvey RM, Enson Y, Ferrer MI. A reconsideration of the origins of pulmonary hypertension. Chest 1971; 59: $82-94$.

11 Naeije R, Vachiery JL, Yerly P, et al. The transpulmonary pressure gradient for the diagnosis of pulmonary vascular disease. Eur Respir J 2013; 41: 217-223.

12 Viles PH, Shepherd JT. Evidence for a dilator action of carbon dioxide on the pulmonary vessels of the cat. Circ Res 1968; 22: 325-332.

13 Brimioulle S, Lejeune P, Vachiery JL, et al. Effects of acidosis and alkalosis on hypoxic pulmonary vasoconstriction in dogs. Am J Physiol 1990; 258: H347-H353.

14 Naeije R, Vanderpool R. Pulmonary hypertension and chronic mountain sickness. High Alt Med Biol 2013; 14: $117-125$.

15 Sime F, Peñaloza D, Ruiz L. Bradycardia, increased cardiac output, and reversal of pulmonary hypertension in altitude natives living at sea level. Br Heart J 1971; 33: 647-657.

16 Hickling KG. Permissive hypercapnia. Respit Care Clin N Am 2002; 8: 155-169.

17 Epstein SK, Singh N. Respiratory acidosis. Respir Care 2001; 46: 366-383.

18 Palange P. Renal and hormonal abnormalities in chronic obstructive pulmonary disease (COPD). Thorax 1998; 53: 989-991.

19 Castro-Añón O, Golpe R, Pérez-de-Llano LA, et al. Haemodynamic effects of non-invasive ventilation in patients with obesity-hypoventilation syndrome. Respirology 2012; 17: 1269-1274. 
20 Sun XG, Hansen JE, Oudiz R, et al. Exercise pathophysiology in patients with primary pulmonary hypertension. Circulation 2001; 104: 429-435.

21 Deboeck G, Niset G, Lamotte M, et al. Exercise testing in pulmonary arterial hypertension and in chronic heart failure. Eur Respir J 2004; 23: 747-751.

22 Groepenhoff H, Overbeek MJ, Mulè M, et al. Exercise pathophysiology in patients with chronic mountain sickness. Chest 2012; 142: 877-884.

23 Peacock AP. Pulmonary hypertension due to chronic hypoxia. BMJ 1990; 300: 763. 